\section{Les coronavirus, ennemis incertains}

Dylan Juckel, Jean Dubuisson, Sandrine Belouzard

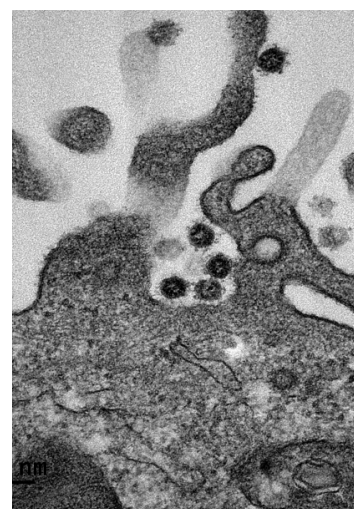

Virologie moléculaire et cellulaire des coronavirus, Centre d'infection et d'immunité de Lille, Institut Pasteur de Lille, Université de Lille, CNRS, Inserm, CHRU, 59000 Lille, France. sandrine.belouzard@ibl.cnrs.fr jean.dubuisson@ibl.cnrs.fr

\section{2: le SARS-CoV}

Cependant, la situation à changé à la fin de l'année 2002, à la suite de l'émergence du premier coronavirus humain hautement pathogène, le SARS-CoV (severe acute respiratory syndrome coronavirus), responsable du syndrome respiratoire aigu sévère (SRAS). Ce nouveau virus a émergé dans la province du Guangdong dans le sud-est de la Chine. En juin 2003, à la fin de l'épidémie qu'il avait entraînée et qui avait été contenue grâce à la mise en place de mesures de quarantaine, 8000 cas de SRAS avaient été déclarés à l'Organisation mondiale de la santé (OMS) à travers le monde, marqués par 800 décès.

Rapidement, la question de l'origine de ce virus s'est posée, et son origine zoonotique, c'est-à-dire sa transmission à partir d'un réservoir animal, fut montrée. Une espèce de chauve-souris de la famille des Rhinolophidae fut ainsi identifiée comme étant l'hôte réservoir du SARS-CoV, grâce à la détection d'anticorps spécifiques de ce coronavirus émergent, par des analyses sérologiques réalisées chez l'animal. Des civettes palmistes (Paguma larvata) provenant d'un marché animalier furent également testées positives par des tests sérologiques détectant des anticorps contre le SARS-CoV, alors que d'autres, sauvages ou issues d'un élevage, se révélèrent négatives dans ces tests. La civette ne pouvait donc pas être un hôte réservoir naturel pour le virus, mais elle fut alors considérée comme un hôte intermédiaire, dans lequel le virus s'était adapté, devenant capable de passer dans l'espèce humaine. C'est donc cet hôte intermédiaire, infecté par des chauves-souris, qui a transmis le virus à l'homme en 2002. On parle dans ce cas d'une transmission zoonotique [2].

\section{Le MERS-CoV}

Dix ans plus tard, un nouveau coronavirus, le MERS-CoV (Middle-east respiratory syndrome coronavirus), fait son apparition. II apparaît dans la péninsule arabique. II s'agit d'un virus qui s'avère plus dangereux que le SARS-CoV de 2002, avec un taux de mortalité estimé à $35 \%$ chez l'homme. Le MERS-CoV s'attaque aux voies respiratoires. II peut provoquer de la fièvre et de la toux, ainsi que de graves pneumonies pour
Vignette (microscopie électronique d'une cellule infectée par le SARS-CoV-2 (๔) Philippe Roingeard et Sébastien Eymieux, unité Inserm U1259, Morphogenèse et antigénicité du VIH et des virus des hépatites, Université de Tours, Tours, France). 
les cas les plus sévères. Les personnes qui présentent des insuffisances cardiovasculaires ou rénales et les personnes immunodéprimées ou diabétiques, sont plus à risque de développer une forme grave de la maladie. Le foyer épidémique était essentiellement situé au Moyen-Orient, mais 27 pays ont déclaré des cas d'infection par le MERS-CoV à l'OMS ; il s'agissait pour la plupart des patients de cas importés. Ainsi, en 2015, un voyageur de retour du Moyen-Orient a été à l'origine d'une épidémie de 185 cas en Corée du Sud, révélant l'importance, alors, de la détection rapide des cas d'infection après un séjour dans la péninsule arabique.

Contrairement au SARS-CoV, le MERS-CoV circule toujours. II a infecté environ 2500 personnes, provoquant la mort de plus de 800 d'entre elles. Le faible nombre de cas déclarés, comparé à celui provoqué par le SARSCoV, s'explique par une mauvaise adaptation ${ }^{1}$ du virus à l'homme, rendant la transmission interhumaine plus difficile. Comme pour les civettes palmistes dans le cas du SARS-CoV, des anticorps spécifiques du MERS-CoV ont été mis en évidence dans le sérum de dromadaires, révélant le possible rôle de cet animal dans la transmission. La circulation de souches de MERS-CoV, identiques chez l'homme et le dromadaire, a également été montrée. Dans le cas de ce virus, la transmission inter-espèces a été rendue possible par les contacts rapprochés entre les hommes et les dromadaires, mais aussi par la consommation de produits provenant de ces animaux, comme le lait, très prisé dans les pays arabes. Le MERS-CoV ne se transmet pas facilement d'homme à homme. Des mesures de quarantaine permettent de contenir facilement sa transmission. II est néanmoins probable que le virus ait été régulièrement introduit dans la population humaine par les dromadaires. Des virus proches du MERS-CoV (en anglais, MERS-like CoV) ont été identifiés chez des chauves-souris, suggérant de nouveau une transmission zoonotique, d'abord des chauve-souris vers le dromadaire, puis du dromadaire à l'homme [2]. Notons que le virus circule chez les dromadaires depuis de nombreuses années et que les dromadaires présents dans le nord de l'Afrique sont également infectés par le MERS-CoV. Pourtant, seules les souches de virus circulant chez les dromadaires du Moyen-Orient sont transmissibles à l'homme.

\section{Le SARS-CoV-2}

À la fin de l'année 2019, plusieurs cas de pneumonies atypiques sévères sont recensés dans la ville de Wuhan, dans la province du Hubei en République Populaire de Chine. Déclarés à l'OMS par les autorités chinoises, l'émergence d'un nouveau coronavirus, similaire au SARS-CoV de 2002, est alors suspectée. Après isolement de la souche infectieuse chez des patients, l'hypothèse d'un nouveau coronavirus est confirmée : il s'agit d'un coronavirus qui partage $80 \%$ de son génome avec celui du SARSCoV. Initialement appelé nCoV-2019, il sera finalement rebaptisé SARSCoV-2 par le Comité international de taxonomie des virus, et la maladie qu'il induit appelée COVID-19 (coronavirus disease 19, 19 pour 2019). Cette maladie émergente, qualifiée de pandémie le 11 mars 2020 par le directeur général de l'OMS, provoque une crise sanitaire et économique sans précédent depuis plusieurs décennies.

Un marché de fruits de mer de la ville de Wuhan semble être l'épicentre de cette nouvelle épidémie. Les premiers cas de COVID-19 ont en effet

${ }^{1}$ Le processus d'adaptation repose en particulier sur la plasticité du génome viral (pour revue voir [21]). tous été identifiés chez des personnes ayant fréquenté ce marché dans les jours qui ont précédé l'apparition des signes de leur infection. Les autorités prennent rapidement la décision de sa fermeture. Dans ce type de marchés, très nombreux en Chine, tous types d'animaux, vivants ou morts, y sont exposés, permettant une forte promiscuité entre les espèces, un facteur favorable à la transmission des agents pathogènes entre les animaux. Néanmoins, encore aujourd'hui, l'origine du virus n'a pas été clairement identifiée, mais l'origine zoonotique est très fortement suspectée : le virus le plus proche du SARS-CoV-2 identifié à ce jour est un virus de chauve-souris qui présente une similarité de génome de $98 \%$ environ, et le pangolin est soupçonné d'être l'hôte intermédiaire.

Au 12 juin 2020, 7558687 cas d'infection, confirmés par des tests spécifiques (identification de la présence du virus par RT-PCR [reverse transcriptase-polymerase chain reaction]) ou après hospitalisation pour détresse respiratoire, ont été recensés à travers le monde (COVID19 Dashboard, Center for Systems Science and Engineering [CSSE], Johns Hopkins university, Baltimore, ÉtatsUnis) ${ }^{2}$, dont au moins 422544 décès, donnant un taux de mortalité moyen d'environ 5,6\%. Ce chiffre (décès/ diagnostiqués positifs) est sans doute surestimé car un grand nombre de cas asymptomatiques n'entrent pas dans ce calcul, puisqu'ils n'ont pas été confirmés par un test diagnostic. Le taux réel de mortalité devrait ainsi être plus faible. À noter que la mortalité varie énormément en fonction des tranches d'âge, les personnes de plus de 65 ans étant les plus à risque de développer une forme sévère de la maladie [3].

\section{Les coronavirus}

Découverts au cours des années 1960 , les coronavirus appartiennent à l'ordre des Nidovirales et plus particulièrement à la famille des Coronaviridae. Ces virus sont retrouvés majoritairement chez les oiseaux et les mammifères (Tableau I). Cette famille est composée de quatre genres: les Alpha-, Beta-, Gamma- et Deltacoronavirus. Les coronavirus sont des virus à ARN simple brin positif ${ }^{3}$ enveloppés (Figure 1). Ils ont la particularité de posséder le plus long génome à ARN parmi les virus à $A R N$, constitué de 27000 à 32000 bases (soit un génome 100000 fois plus petit que le génome humain). Ce génome est coiffé en $5^{\prime 4}$ et polyadénylé en 3 '. Les

\footnotetext{
2 https://gisanddata.maps.arcgis.com/apps/opsdashboard/index.html\#/ bda7594740fd40299423467b48e9ecf6

${ }^{3}$ La séquence des gènes sur l'ARN viral génomique est identique à celle des ARN messagers.

${ }^{4}$ La coiffe, constituée par ajout d'une guanosine, protège les ARN de la dégradation
} par les nucléases cellulaires. 


\begin{tabular}{|c|c|c|}
\hline Genre & Sous-genre & Espèce \\
\hline \multirow{16}{*}{ 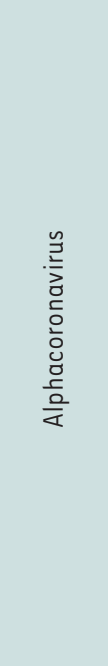 } & Colacovirus & Coronavirus de chauve-souris CDPHE15 \\
\hline & \multirow[t]{2}{*}{ Decacovirus } & Coronavirus de chauve-souris HKU1O \\
\hline & & Alphacoronavirus de chauve-souris Grand rhinolophe (HuB-2013) \\
\hline & Duvinacovirus & Coronavirus humain HCoV-229ع \\
\hline & Luchacovirus & Coronavirus de rat \\
\hline & Minacovirus & Coronavirus de vison et de furet \\
\hline & Minunacovirus & Coronavirus de chauve-souris Miniopterus (HKU8) \\
\hline & Myotacovirus & Coronavirus de chauve-souris Myotis ricketti Sax-2011 \\
\hline & Nyctacovirus & Coronavirus de chauve-souris Nyctalus velutinus SC-2013 \\
\hline & \multirow[t]{2}{*}{ Pedacovirus } & Virus de la diarrhée épidémique porcine (PEDV) \\
\hline & & Coronavirus de chauve-souris Scotophilus 512 \\
\hline & Rhinacovirus & Coronavirus de chauve-souris Rhinolophus HKU2 \\
\hline & \multirow[t]{2}{*}{ Setracovirus } & Human coronavirus NL63 \\
\hline & & Coronavirus de chauve-souris proche de NL63 (BtKYNL63-9b) \\
\hline & \multirow[t]{2}{*}{ Tegacovirus } & Alphacoronavirus I (virus félin et canin) \\
\hline & & Virus de la gastroentérite transmissible (TGEV, virus de porc) \\
\hline \multirow{11}{*}{ 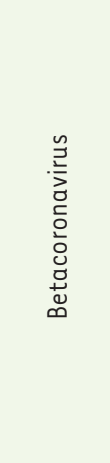 } & \multirow[t]{5}{*}{ Embecovirus } & Betacoronavirus 1 (coronavirus humain $0 \mathrm{C} 43$ et coronavirus bovin) \\
\hline & & Coronavirus humain $\mathrm{HKUl}$ \\
\hline & & Coronavirus murin (MHV, mouse hepatitis virus) \\
\hline & & China Rattus coronavirus HKU24 \\
\hline & & Bat Hp-betacoronavirus/Zhejiang2013 \\
\hline & Hibecovirus & coronavirus de hérisson 1 \\
\hline & \multirow[t]{3}{*}{ Merbecovirus } & Virus du syndrome respiratoire du Moyen-Orient (MERS-CoV) et virus proches (humain, dromadaire, chauve-souris) \\
\hline & & Coronavirus de chauve-souris Pipistrellus HKU5 \\
\hline & & Coronavirus de chauve-souris Tylonycteris HKU4 \\
\hline & Nobecovirus & Coronavirus de chauve-souris roussettes (HKU9 et GCCDCl) \\
\hline & Sarbecovirus & Coronavirus du SARS et virus proches du SARS \\
\hline \multirow{5}{*}{ 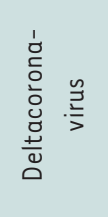 } & Andecovirus & Coronavirus de canard (HKU20) \\
\hline & Buldecovirus & Coronavirus de porc HKU15 \\
\hline & & Coronavirus de passereaux (HKU13 et HKU16) \\
\hline & Herdecovirus & Coronavirus des bihoreaux (HKU19) \\
\hline & Moordecovirus & Coronavirus des poules d'eau (HKU21) \\
\hline \multirow{2}{*}{ 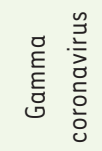 } & Cegacovirus & Coronavirus du béluga (SWl) \\
\hline & Igacovirus & Coronavirus aviaires infectant les poulets, pigeons, dindes \\
\hline
\end{tabular}

Tableau I. Les différents coronavirus (liste non exhaustive).

coronavirus sont des virus sphériques, d'environ $100 \mathrm{~nm}$, enveloppés d'une bicouche lipidique dans laquelle sont ancrées différentes protéines (voir plus loin). Tout comme l'ADN génomique présent dans le noyau de chacune de nos cellules, I'ARN génomique des coronavirus porte l'information génétique indispensable à la production de nouveaux virions [4]. Plus d'une dizaine de cadres de lecture (en anglais open reading frame, ORF) constituent le génome viral (Figure 2). Deux ORF dénommées ORFla et ORFlb, constituant les deux tiers de ce génome, codent 2 polyprotéines, ppla et pplab. Ces deux ORF présentent un unique codon d'initiation et de terminaison. Elles ne constituent par conséquent qu'un seul et unique cadre de lecture. La biosynthèse des deux polyprotéines est rendue possible grâce à un décalage du cadre de lecture causé par une structure secondaire particulière de l'ARN génomique, un pseudonœud, qui provoque le retour en arrière d'une base sur le ribosome. Ppla et pplab sont les précurseurs de 15 à 16 protéines, dites non-structurales, que l'on nomme nsp (non-structural proteins), qui seront produites par auto-clivage des deux précurseurs. Les protéines ainsi produites s'assemblent ensuite entre elles pour former le complexe réplicase-transcriptase indispensable à la réplication et à la transcription de I'ARN génomique viral lors de l'infection. Chacune des protéines nsp possède une activité précise : nspl2 est ainsi responsable 


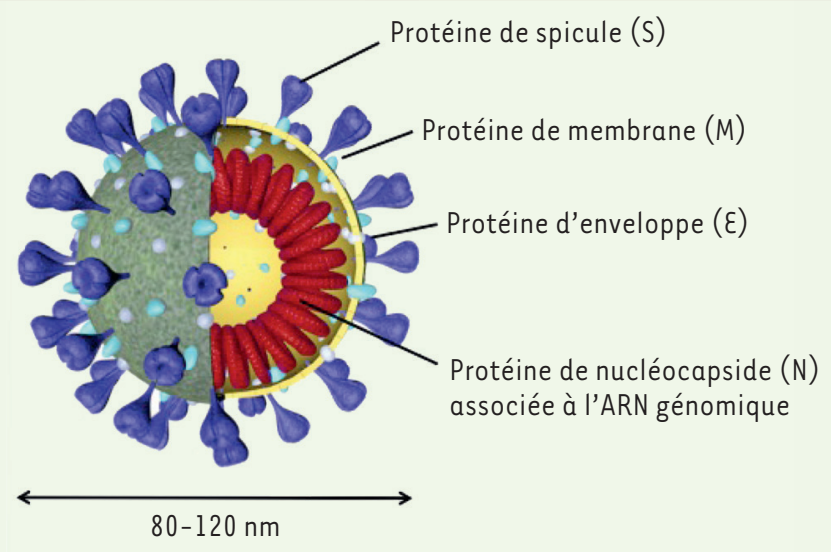

Figure 1. Représentation schématique d'un coronavirus.

de l'activité polymérase dépendante de l'ARN et nspl4, de l'activité de relecture, permettant de corriger de potentielles erreurs insérées lors de la synthèse d'un nouveau brin d'ARN viral [5]. Le tiers restant du génome code quatre protéines structurales: $S, \varepsilon, M$ et $N$. Certains coronavirus en possèdent une cinquième: l'hémagglutinine estérase $(H \varepsilon)$. Des gènes codant des protéines «accessoires » (nommés ORF3, ORF4, etc.), en nombre variable, sont également insérés entre les gènes codant les protéines structurales. Bien que la majorité des fonctions de ces protéines accessoires soient encore aujourd'hui mal comprises, elles semblent jouer un rôle très important dans la pathogénicité des coronavirus. Une étude réalisée sur des virus mutants, n'exprimant qu'une partie de ces protéines, révèlent en effet leur implication dans l'atténuation de la réponse immunitaire innée de l'hôte, en inhibant la synthèse des interférons de type $\beta$ [6], essentiels dans la lutte contre une infection virale.

\section{Les protéines structurales}

La première protéine structurale, la protéine Spike (S), est une protéine de 180-200 kDa, très fortement glycosylée, qui s'assemble sous forme de trimères et est ancrée à la surface de l'enveloppe virale. C'est cette protéine qui donne cette apparence en forme de couronne aux coronavirus en microscopie électronique. Elle est constituée de deux domaines, $\mathrm{SI}$ et $\mathrm{S} 2$, et permet à la particule virale de se fixer à un récepteur membranaire exprimé par la cellule hôte afin d'engager son entrée dans cette dernière. La liaison au récepteur cellulaire repose sur le domaine Sl de la protéine $S$, qui contient un domaine de liaison au récepteur (receptor-binding domain, RBD). Le domaine S2 est, quant à lui, un domaine de fusion nécessaire pour la fusion de l'enveloppe virale avec la membrane de la cellule.

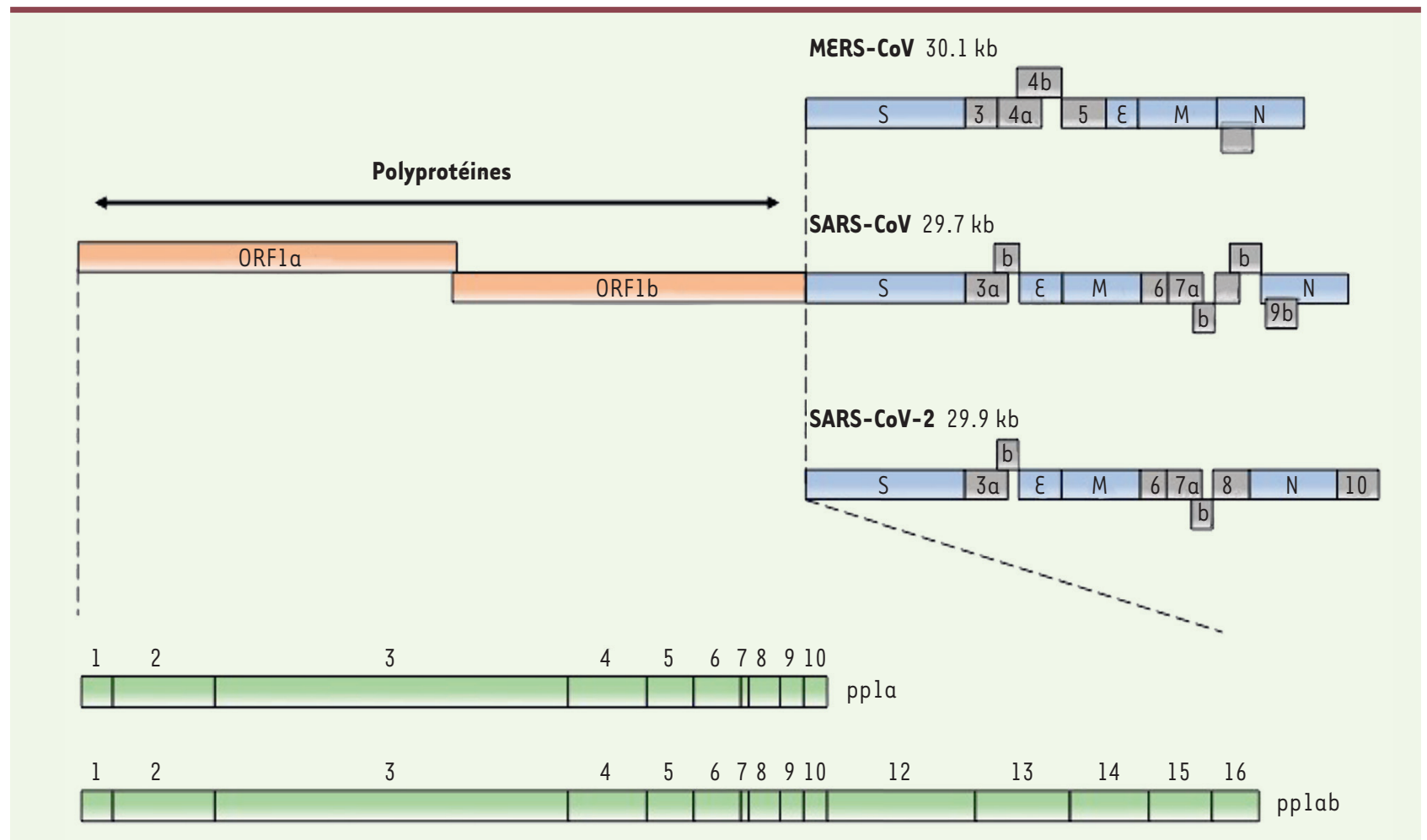

Figure 2. Organisation schématique du génome des coronavirus. Les deux premiers tiers du génome (orange) codent les polyprotéines ppla et pplab. Ces polyprotéines (vert) sont à l'origine de 16 protéines non structurales qui sont générées par clivage protéolytique. Le dernier tiers du génome code les protéines structurales (en bleu) et les protéines accessoires (en gris). 
La seconde protéine structurale, codée par le génome, est la protéine d'enveloppe $\varepsilon$. Petite protéine de 8 à 12 kDa, elle est la moins abondante des protéines de l'enveloppe virale, mais elle n'est pourtant pas la moins importante. Elle se compose en $\mathrm{N}$-terminal d'un court ectodomaine hydrophile, d'un long et unique domaine transmembranaire et d'un segment hydrophile en C-terminal, formant l'endodomaine. La découverte d'une activité de canal ionique suggère que cette protéine intervient dans la sécrétion des nouveaux virions. Cette protéine semble également indispensable à l'assemblage de l'enveloppe virale. $\varepsilon$ n effet, plusieurs études ont montré que la protéine $\varepsilon$, ainsi que la protéine $M$, étaient nécessaires pour produire des particules sub-virales, c'est-à-dire des particules dépourvues de génome et qui ont les mêmes taille et forme qu'une particule virale complète [7].

La protéine membranaire $M$ est la troisième protéine structurale codée par le génome. Elle est la plus abondante des protéines constituant l'enveloppe virale. Protéine de 25 à $35 \mathrm{kDa}$, elle possède au moins un site de glycosylation ( 0 - ou $\mathrm{N}$-glycosylation selon le genre du virus) localisé au niveau d'un très court ectodomaine. Ce domaine est suivi de trois domaines transmembranaires, eux-mêmes suivis d'une longue séquence $\mathrm{C}$-terminale qui représente plus de la moitié de la protéine. La protéine M est considérée comme le moteur de l'assemblage des particules virales, en raison des diverses interactions intermoléculaires qu'elle établit avec les autres protéines ( $M-S$, $M-\varepsilon, M-N, M-M)$, les interactions $M-M$ et $M-\varepsilon$ étant les plus importantes pour l'assemblage.

La quatrième et dernière protéine structurale est la protéine de nucléocapside $(\mathrm{N})$, une phosphoprotéine de 43-50 kDa associée à l'ARN génomique pour former la nucléocapside hélicoïdale [8].

\section{Le cycle viral}

Le cycle du virus dans la cellule se décompose en trois grandes étapes: (1) l'entrée du virus dans la cellule hôte, (2) la réplication du génome et (3) la formation et la sécrétion de nouveaux virions (Figure 3).

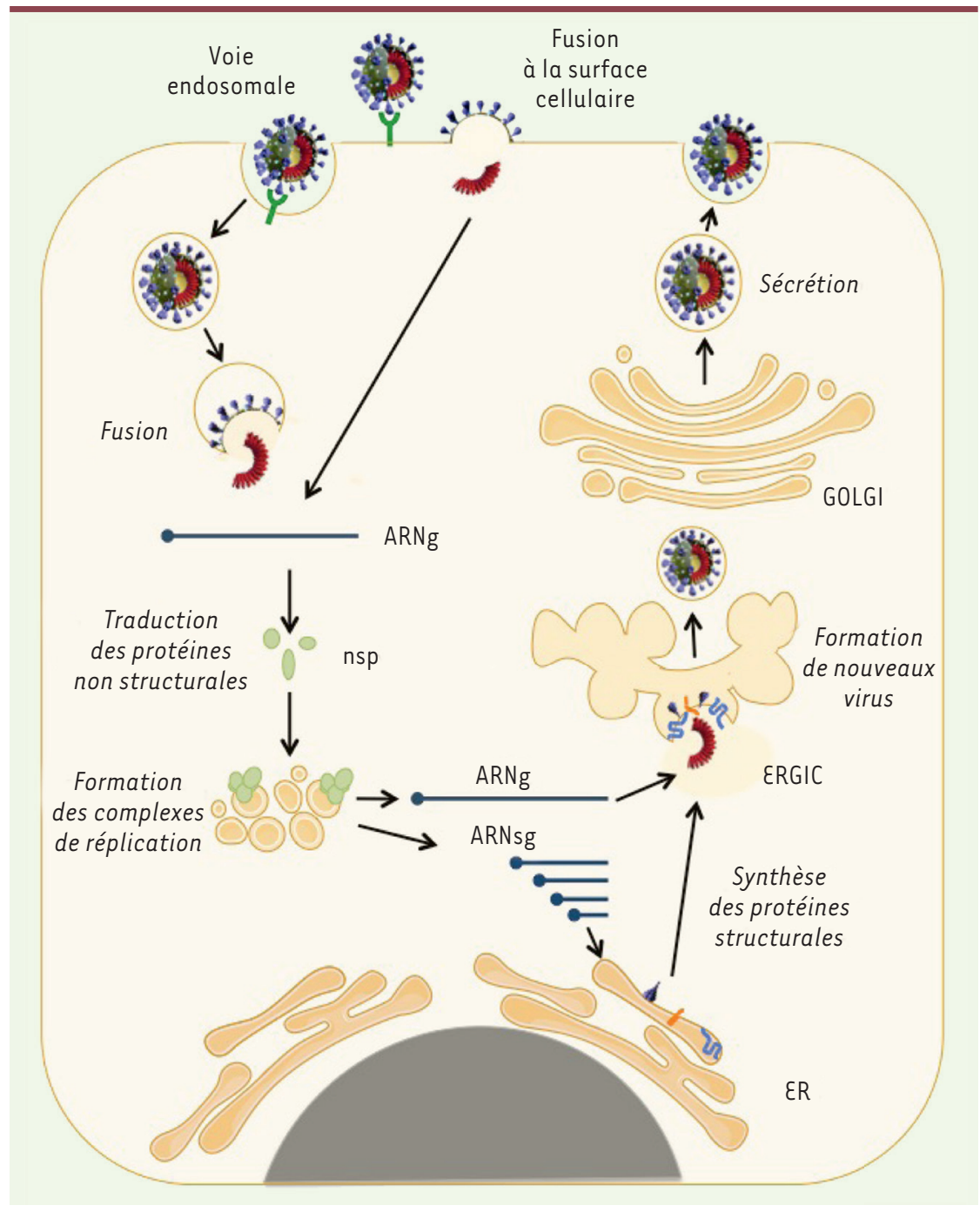

Figure 3. Cycle viral des coronavirus. L'entrée du virus dans la cellule constitue la première étape du cycle viral. Le virus lie un récepteur à la surface des cellules (en vert, DPP4 pour le MERS-CoV ou ACE2 pour les SARS-CoV et SARS-CoV-2). Le virus libère son génome dans la cellule cible après fusion de son enveloppe lipidique avec une membrane de celle-ci. $\varepsilon n$ fonction des protéases présentes, cette fusion peut avoir lieu directement avec la membrane de la surface cellulaire ou dans des endosomes après internalisation du virus. Le génome est ensuite traduit pour produire les protéines non-structurales qui vont former les complexes de réplication et induire des réarrangements de membrane. La réplication du génome va permettre la formation de nouveaux ARN génomiques ( $\mathrm{ARNg}$ ) qui pourront être incorporés dans les particules virales néo-synthétisées. Les protéines de structure du virus ( $S, \varepsilon, M$ et $N$ ) sont produites à partir des ARN sous-génomiques (ARNsg). Les nouvelles particules virales sont assemblées au niveau du compartiment intermédiaire entre le réticulum endoplasmique et le Golgi (ERGIC). Elles sont ensuite sécrétées dans le milieu extracellulaire. 


\section{L'entrée virale}

L'entrée du virus regroupe toutes les étapes allant de son contact avec la cellule hôte jusqu'à la libération de son génome à l'intérieur de la cellule. La capacité des coronavirus, comme pour les autres virus, à entrer dans une cellule hôte pour l'infecter, repose sur la reconnaissance d'un récepteur que celle-ci exprime à sa surface. Dans le cas des coronavirus, c'est la protéine $S$ qui est responsable de la reconnaissance du récepteur cellulaire, souvent une protéine ayant une activité enzymatique. Dans le cas des virus SARS-CoV et SARS-CoV-2, la protéine $S$ utilise comme récepteur l'enzyme de conversion de l'angiotensine 2, ACE2 (angiotensin-converting enzyme 2) [9] $(\rightarrow)$ exprimée, entre autres, à la surface des cellules pulmonaires, des pneumocytes de type II et des macrophages alvéolaires. ACE2 $(\rightarrow)$ Voir la Nouvelle de J.J. Mercadier, $\mathrm{m} / \mathrm{s} \mathrm{n}^{\circ} 2$, février 2003, page 141 est aussi exprimée par les cellules endothéliales vasculaires du cœur, et intervient dans la régulation de la pression sanguine $^{5}$, ce qui pourrait expliquer le plus haut risque des personnes souffrant de maladies cardio-vasculaires, comme l'hypertension, en cas de COVID-196. Dans le cas du MERS-CoV, la protéine $S$ cible spécifiquement le récepteur DPP4 (dipeptidyl peptidase-4), ou CD26, une protéine impliquée dans la transduction du signal ou l'apoptose ${ }^{7}$. Plus précisément, comme pour le SARS-CoV-2, c'est le RBD contenu dans le domaine S1 de la protéine qui porte l'activité de liaison au récepteur. Ainsi, pour délivrer son génome, le virus doit fusionner son enveloppe virale avec la membrane de la cellule cible. Pour assurer le succès de l'infection, cette fusion nécessite d'être finement orchestrée, avec, tout d'abord, la délivrance d'un signal d'activation donné au virus. La fusion est ainsi initiée à la suite d'importants réarrangements conformationnels de la protéine $S$ qui résultent de son clivage protéolytique par des protéases de la cellule cible. Comme l'hémagglutinine du virus de la grippe, la protéine $S$ est une protéine de fusion de type I. Elle présente un peptide (dit peptide de fusion) qui, au cours des réarrangements conformationnels de la protéine, est inséré dans la membrane de la cellule cible. Les modifications conformationnelles de la protéine qui s'ensuivent permettent alors le rapprochement et la fusion de l'enveloppe virale avec la membrane cellulaire, ce qui provoque la formation d'un pore par lequel la nucléocapside, contenant le génome viral, est injectée dans le cytoplasme de la cellule. Ces modifications de la protéine $S$ sont essentielles pour exposer le peptide de fusion ; les coronavirus utilisent différentes protéases cellulaires pour réaliser ces modifications, multipliant ainsi les possibilités d'activation de la fusion pour garantir la réussite de l'infection. Selon la protéase utilisée, deux voies d'entrée s'offrent au virus. Si certaines protéases sont exprimées à la membrane de la cellule, comme la protéase TMPRSS2 (transmembrane serine protease 2), la fusion sera réalisée directement à la surface de la cellule. Mais en l'absence de telles protéases, le virus sera internalisé dans le cytoplasme par endocytose. L'activation de la fusion

${ }^{5}$ L’ACદ2 régule négativement le système rénine-angiotensine et agit comme une protéine de clairance de l'angiotensine II, qui a des effets vasoconstricteur, pro-fibrosant, et pro-inflammatoire.

${ }^{6}$ L'ARN codant les protéines virales a également été retrouvé dans les cellules productrices de mucus dans les fosses nasales, et dans les cellules intestinales.

${ }^{7}$ Le récepteur DPP4 intervient dans le métabolisme du glucose. Elle dégrade les incrétines (glucagon-like peptide-1 [GLPl] et gastric inhibitory polypeptide [GIP]). entre le virus et l'endosome (la vésicule d'endocytose) qui le contient fera alors intervenir les protéases endosomales, comme les cathepsines ou la furine [10].

\section{La réplication virale}

La fusion aboutit finalement à la libération de l'ARN génomique dans le cytoplasme de la cellule infectée. Cet ARN servira de matrice, utilisant la machinerie de traduction de la cellule, pour synthétiser les deux polyprotéines ppla et pplab qui formeront le complexe réplicase-transcriptase après leur maturation. Ppla code les protéines nspl à nspll, et pplab, les protéines nspl à nspl6. Ces deux polyprotéines sont clivées grâce aux activités protéasiques de deux protéines différentes, nsp3 et nsp5, produites par autoclivage. Nsp3 clive les jonctions nspl-2, nsp2-3 et nsp3-4, tandis que nsp5 clive toutes les jonctions des protéines suivantes. Le complexe réplicase-transcriptase initie ensuite les étapes de réplication successives de l'ARN génomique. Les réplicons ainsi constitués seront encapsulés dans les futurs virions. Le complexe réalisera également la transcription de l'ensemble des ORF codant les protéines structurales et accessoires, sous la forme d'ARN messagers (ARNm) dits «sous-génomiques ». Le complexe réplicasetranscriptase est ancré dans un réseau membranaire de la cellule infectée, appelé réseau vésiculo-membranaire, qui est dérivé de son réticulum endoplasmique et qui forme des « usines » d'amplification virale [11]. Ce réseau est probablement important pour concentrer les éléments viraux et cellulaires nécessaires à la réplication du virus, mais aussi pour créer un environnement protecteur contre les défenses intracellulaires de l'hôte. La réplication de l'ARN génomique repose sur la synthèse initiale d'un intermédiaire ARN négatif qui sert ensuite de matrice pour la synthèse des ARN génomiques de pleine longueur. Cette synthèse intermédiaire est réalisée par nsp12, une polymérase à ARN dépendante de l'ARN. Contrairement à la réplication de I'ARN génomique qui est un processus de synthèse continue, le mécanisme de transcription est discontinu et caractéristique des Nidovirus. II aboutit à la synthèse d'ARN sous-génomiques qui codent les protéines accessoires et structurales. Ces ARNm sous-génomiques seront ensuite traduits par la machinerie ribosomale de la cellule hôte.

\section{L'assemblage et la sécrétion des virions}

Les protéines $S$, $M$ et $\varepsilon$, traduites simultanément, sont ensuite adressées au réticulum endoplasmique de la cellule. Des groupements glycosides sont alors ajoutés aux protéines natives $S$ et $M$. Celles-ci seront ensuite transportées, par voie vésiculaire, vers le compartiment intermédiaire entre le réticulum endoplasmique et l'appareil de Golgi (ERGIC), qui est le site d'assemblage des coronavirus. C'est le lieu d'une multitude d'interactions protéiques et du bourgeonnement 
des nouvelles particules virales. Cette étape dans le ERGIC est cruciale pour le bon assemblage des virions. Les virions néoformés seront ensuite sécrétés par exocytose afin d'infecter les cellules environnantes, voire un nouvel hôte, par contamination via les gouttelettes de sécrétions oropharyngées dispersées par la toux d'une personne infectée, ou manuportés.

\section{Les pistes thérapeutiques}

En raison de l'arrêt spontané de l'épidémie de SRAS due au SARS-CoV en 2002, sans qu'il n'ait été besoin de traitement particulier, et du faible taux de transmission du MERS, aucun traitement antiviral ou vaccin n'a fait l'objet de développement, faute d'intérêt des institutions de recherche nationales et d'organisations internationales et d'investissement par les compagnies pharmaceutiques des pays développés. II est donc actuellement difficile de prendre en charge les patients les plus graves atteints du COVID-19. Face à cette absence de traitement et de vaccin, de très nombreux pays ont pris la décision en 2020, comme lors des épidémies qui ont jalonné l'Histoire humaine, de confiner leur population en les invitant à respecter certaines consignes d'hygiène (lavage régulier des mains, distanciation, port de masques dans les pays d'Asie, et, plus tardivement, dans les autres pays, etc.), seuls remparts disponibles à la propagation du virus. Certains travaux de recherche sur les traitements anti-coronavirus avaient néanmoins été poursuivis. Plusieurs molécules, dont l'activité anti-coronavirus ou d'autres types de virus avait été démontrée, font aujourd'hui l'objet de nombreux essais cliniques afin de trouver au plus vite une solution thérapeutique contre le COVID-19, afin, en particulier, de désengorger les services de réanimation et ainsi de sauver un maximum de vies.

Il s'agit de molécules HTA (host-targeting antiviral) ciblant indirectement le virus, comme la chloroquine, qui interférerait avec le cycle du virus en altérant possiblement l'étape d'entrée par endocytose du virus, ou de molécules qui affectent directement le virus [22], les DAA (direct-acting antiviral), en interférant avec son assemblage ou sa réplication. D'autres molécules ciblent les conséquences, notamment inflammatoires, de l'infection qui sont à l'origine des détresses respiratoire observées. Toutes ces molécules HTA et DAA font l'objet d'essais intégrant des cohortes de patients plus ou moins importantes.

\section{L'essai clinique européen Discovery coordonné par l'Inserm et l'essai international Solidarity coordonné par l'OMS}

Quatre molécules candidates anti-virales ont été initialement intégrées dans deux essais cliniques de grande ampleur, Discovery, un essai clinique européen randomisé ouvert, mené avec des patients atteints de formes sévères du COVID-19, coordonné par l'Inserm dans le cadre du consortium Reacting ${ }^{8}$, et Solidarity, un essai clinique international randomisé lancé par l'OMS, associant plus de 70 pays (au 27 mars 2020) dont la France ${ }^{9}$. Les premiers résultats ne sont encore

${ }^{8}$ https://www.inserm.fr/actualites-et-evenements/actualites/covid-19-demarrage-essai-cliniquediscovery

${ }^{9}$ https://www.who.int/fr/emergencies/diseases/novel-coronavirus-2019/global-research-on-novelcoronavirus-2019-ncov/solidarity-clinical-trial-for-covid-19-treatments pas publiés ${ }^{10}$. Cependant, fin mai 2020 , le bras de l'essai correspondant au traitement par l'hydroxychloroquine a été arrêté dans ces deux essais.

Ces essais, menés dans le respect des règles encadrant les protocoles de recherche clinique et de l'éthique des essais cliniques $[12,13](\rightarrow)$, visent à évaluer le bénéfice thérapeutique de différentes molécules et leur sécurité d'utilisation (modalités de traitements : soins standards + $(\rightarrow)$ Voir l’éditorial de A. Alperovitch, P. Lazar, $\mathrm{m} / \mathrm{s} \mathrm{n}^{\circ} 4$, avril 2020, page 303 , et le Forum de P. Amiel et al., $\mathrm{m} / \mathrm{s} \mathrm{n}^{\circ} 5$, mai 2020, page 521 molécules testées par rapport à la modalité de traitement dite «soins standards »). Ces molécules ont été les suivantes jusqu'à fin mai 2020 :

Le remdésivir, une molécule initialement développée pour lutter contre le virus Ebola [14] est un candidat dans la lutte contre le SARS-CoV-2. C'est un analogue nucléotidique monophosphate de l'adénosine. Lors de la réplication virale, il agit comme un leurre pour la polymérase virale et est incorporé au sein des réplicons. L'information génétique alors rendue erronée par sa présence, il se produit un ralentissement, voire un blocage de la synthèse de l'ARN viral. Le cycle viral des nouveaux virions est ainsi compromis ou avorté. Une étude pilotée par le National Institute of Allergy and Infectious Diseases (NIAID) des National Institutes of Health (NIH) (étude ACT, adaptative Covid-19 treatment trial) a montré, lors d'une analyse intermédiaire faite le 29 avril, un bénéfice de cette molécule chez des patients atteints de formes sévères du COVID-19, portant sur la réduction du temps nécessaire à l'amélioration de leur état clinique ( 11 jours contre 15 jours pour le groupe «placebo »). Cependant, un premier essai multicentrique randomisé en double aveugle et contrôlé par placebo réalisé en République populaire de Chine a montré que le remdésivir n'est pas associé à des avantages cliniques statistiquement significatifs [15].

Le ritonavir et le lopinavir, autre type d'antiviraux, dont l'association constitue le Kaletra ${ }^{\circledR}$, pourraient également être efficaces contre le SARS-CoV-2. Essentiellement utilisés contre le virus de l'immunodéficience humaine (VIH), leur activité antivirale, par inhibition des protéases du virus [16], a aussi été montrée contre le SARS-CoV de 2002 [17]. Le Kaletra ${ }^{\circledR}$ est en essai, en association ou non avec l'interféron bêta-la, une cytokine ayant une puissante action anti-virale, bien que son mécanisme d'action potentiel contre le SARS-CoV-2 ne soit pas clairement établi.

\footnotetext{
${ }^{10}$ À la date de rédaction de cet article, l'essai Discovery a pris du retard en ce qui concerne le nombre de patients recrutés par certains pays participants. Les patients français acceptant d'entrer dans l'essai demandent aussi, en majorité, d'être inclus dans le bras hydroxychloroquine, ce qui augmente ce retard dans l'inclusion de patients dans les autres bras (https://www.lemonde.fr/ sciences/article/2020/05/01/covid-19-sur-les-essais-cliniques-I-europe-est-unechec_6038383_1650684.html).
} 
Enfin, l'hydroxychloroquine, un dérivé de la chloroquine, prescrit depuis les années 1950 pour traiter les cas de paludisme, dû aux parasites du genre Falciparum et également utilisée pour traiter des patients souffrant de lupus érythémateux disséminé et de polyarthrite rhumatoïde, du fait de son activité anti-inflammatoire. Son effet antiviral a été montré dans des expériences réalisées in vitro, dans des cultures de cellules infectées avec le SARS-CoV. Dans le cas où le virus utilise la voie endosomale ${ }^{11}$, l'hydroxychloroquine inhiberait en effet l'entrée de celui-ci dans la cellule du fait de la modification de $\mathrm{pH}$, normalement acide, qu'elle induit dans les compartiments endosomiaux/lysosomiaux, conduisant à un dysfonctionnement de ces derniers. Cela a été montré avec le virus influenza [18] mais n'a pas été rapporté à ce jour en ce qui concerne le SARS-CoV-2. Son mode d'action est donc différent de celui de remdésivir. Cependant, les coronavirus pouvant également pénétrer dans la cellule par fusion de leur enveloppe avec la membrane plasmique, notamment dans les cellules de l'arbre respiratoire, les capacités de l'hydroxychloroquine à inhiber l'entrée virale sont susceptibles d'être beaucoup moins efficaces in vivo. Dans certains essais cliniques, l'administration d'hydroxychloroquine a été associée à celle d'azythromycine, un antibiotique de la famille des macrolides indiqué dans les infections pulmonaires bactériennes, alors que son mode d'action anti-virale reste non documenté [19]. Seule l'hydroxychloroquine a été testée dans les deux essais sus-mentionnés (Discovery et Solidarity), jusqu'à la suspension de ce bras fin mai 2020 décidé respectivement par l'agence nationale de sécurité du médicament et des produits de santé $(\text { ANSM })^{12}$ et I'OMS. Celle-ci, sur la base des recommandations de son comité chargé de l'innocuité et du suivi des données de l'essai Solidarity, a levé cette suspension le 3 juin $2020^{13}$.

\section{Les autres essais}

Dans l'essai Coviplasm, des équipes de l'hôpital Saint-Antoine - AP-HP, Sorbonne Université, IPLESP UMR-S1136, s'intéressent à un traitement utilisant les anticorps produits par les patients infectés ayant surmonté la maladie, par transfusion de leur plasma aux patients hospitalisés ${ }^{14}$.

En parallèle à la quête d'une thérapie antivirale, plusieurs laboratoires se sont déjà penchés sur la mise au point d'un vaccin contre le SARSCoV-2. Bien qu'il faille patienter encore plusieurs mois avant de voir les premiers résultats de campagnes de vaccination. Enfin, certaines pistes pourraient également se révéler prometteuses, comme celle du BCG, un vaccin antituberculeux qui par sa capacité à stimuler une immunité innée entraînée [20] pourrait induire une protection partielle non spécifique contre le SARS-CoV-2, en augmentant les capacités de la réponse innée. Le tocilizumab, un anticorps monoclonal humanisé spécifique de la chaîne alpha du récepteur de l'interleukine 6 (IL-6R $\alpha)$, qui inhibe la fixation

\footnotetext{
${ }^{11}$ La chloroquine a un effet sur les vésicules endosomales en modifiant leur pH. Elle est utilisée pour inhiber la phagocytose ou l'autophagie, deux mécanismes cellulaires qui nécessitent le bon fonctionnement des endolysomes.

12 https://www.ansm.sante.fr/S-informer/Points-d-information-Points-d-information/COVID-19-IANSM-souhaite-suspendre-par-precaution-les-essais-cliniques-evaluant-I-hydroxychloroquine-dansla-prise-en-charge-des-patients-Point-d-Information

${ }^{13} \mathrm{https}$ ://www.who.int/fr/dg/speeches/detail/who-director-general-s-opening-remarks-at-the-mediabriefing-on-covid-19---03-june-2020

${ }^{14}$ https://www.aphp.fr/contenu/covid-19-demarrage-de-lessai-clinique-coviplasm-visant-tester-lefficacite-de-la-transfusio-0
}

de cette dernière à ce récepteur, a récemment été testé dans le cadre du réseau REACTing au sein de la pateforme CORIMUNO de I'AP-HP. Cet anticorps semble permettre de moduler la réponse inflammatoire exacerbée (orage cytokinique caractérisé notamment par des taux très élevés d'IL-6) induite par le virus et qui détruit les alvéoles pulmonaires.

\section{Conclusion}

Les coronavirus constituent une famille de virus qui sont connus pour leur capacité à franchir les barrières d'espèces et à en infecter de nouvelles. Ainsi nous avons assisté, depuis le début du xxl ${ }^{e}$ siècle, à l'émergence de trois coronavirus très pathogènes pour l'homme, la dernière ayant résulté en une pandémie mondiale avec des conséquences économiques et sociales qui se sont révélées dévastatrices. Une course contre la montre, à l'échelle mondiale, s'est engagée pour faire face à cette pandémie, en développant des traitements et un vaccin. Nous devons néanmoins aussi penser au futur et nous préparer au risque d'épidémies dues à l'émergence de nouveaux coronavirus à transmission zoonotique. Cela nécessite, sans doute, une prise de conscience des modalités permettant le maintien de la barrière entre espèces et, surtout, le développement de traitements qui seraient efficaces contre toute forme de coronavirus, qu'ils soient très ou peu pathogènes pour l'homme, leur risque de transmission et leur dangerosité ne pouvant être, a priori, anticipés. L'identification de cibles thérapeutiques adéquates nécessite donc d'approfondir les connaissances que nous avons du cycle et de la pathogenèse de ces virus. $\diamond$

\section{SUMMARY}

\section{Coronavirus, emerging viruses}

Coronavirus is a large family of viruses that infect mammals and birds. Coronaviruses are known to cross barrier species and infect new ones. In the past twenty years, we witnessed the emergence of three different coronaviruses, the latest one being the SARS-CoV-2 (severe acute respiratory syndrome coronavirus 2) responsible for the COVID-19 (covid disease 19) pandemic. Coronaviruses are enveloped virus with a long positive sense RNA genome. Like all viruses, they hijack the cellular machinery to replicate and produce new virions. There is no approved vaccine or specific antiviral molecule against coronaviruses but with the urgency to treat COVID-19, several candidate therapies are currently investigated. $\diamond$

\section{LIENS D'INTÉRÊT}

Les auteurs déclarent n'avoir aucun lien d'intérêt concernant les données publiées dans cet article. 


\section{RÉFÉRENCES}

1. Vabret A, J. Dina J, Brison $\varepsilon$, et al. Coronavirus humains (HCoV). Pathol Biol 2009 ; 57 : 149-60.

2. De Wit $\varepsilon$, van Doremalen $N$, Falzarano $D$, et al. SARS and MERS: recent insights into emerging coronaviruses. Nat Rev Microbiol 2016 ; 14 : 523-34.

3. Zhu N, Zhang D, Wang W, et al. A novel coronavirus from patients with pneumonia in China, 2019. N Engl J Med $2020 ; 382$ : 929-36.

4. Masters PS. The molecular biology of coronaviruses. Adv Virus Res $2006 ; 66: 193-292$

5. Neuman BW, Chamberlain P, Bowden F, et al. Atlas of coronavirus replicase structure. Virus Res $2014 ; 194: 49-66$

6. Perlman S, Netland J. 2009. Coronaviruses post-SARS: update on replication and pathogenesis. Nat Rev Microbiol $2009 ; 7: 439-50$

7. Bos $\varepsilon C$, Luytjes $W$, Van der Meulen H, Koerten HK, et al. The production of recombinant infectious DI-particles of a murine coronavirus in the absence of helper virus. Virology $1996 ; 218: 52-60$.

8. De Haan CAM, Rottier PJM. Molecular interactions in the assembly of coronaviruses. Adv Virus Res $2005 ; 64: 165-230$.

9. Mercadier JJ. Une seconde enzyme de conversion de l'angiotensine juste pour le cœur ? Med Sci (Paris) $2003 ; 19: 141-3$.

10. Millet JK, Whittaker GR. Host cell proteases: critical determinants of coronavirus tropism and pathogenesis. Virus Res $2015 ; 202: 120$-34.

11. Hagemeijer MC, Rottier PJM, de Haan CAM. Biogenesis and dynamics of the coronavirus replicative structures. Viruses $2012 ; 4: 3245-69$

12. Alperovitch A, Lazar P. L'éthique des essais thérapeutiques. Med Sci (Paris) $2020 ; 36: 303-7$.

13. Amiel P, Chneiweiss H, Dosquet C. Covid-19: protocoles de soins ou protocoles de recherche? Med Sci (Paris) $2020 ; 36: 521-3$

14. Warren TK, Jordan R, Lo MK, et al. Therapeutic efficacy of the small molecule GS-5734 against Ebola virus in rhesus monkeys. Nature 2016 ; $531: 381-5$.
15. Wang Y, Zhang D, Du G, et al. Remdesivir in adults with severe COVID-19: a randomised, double-blind, placebo-controlled, multicentre trial. Lancet 2020 ; doi.org/10.1016/S0140-6736(20)31022-9.

16. Barragan P, Podzamczer D. Lopinavir/ritonavir: a protease inhibitor for HIV-1 treatment. Expert Opin Pharmacother 2008 ; $9: 2363-75$

17. Wu CY, Jan JT, Ma SH, et al. Small molecules targeting severe acute respiratory syndrome human coronavirus. Proc Natl Acad Sci USA 2004 ; $101: 10012-17$.

18. Yan $Y$, Zou Z, Sun Y, et al. Anti-malaria drug chloroquine is highly effective in treating avian influenza $A \mathrm{H} 5 \mathrm{Nl}$ virus infection in an animal model. Cell Res $2013 ; 23: 300-2$.

19. Gautret P, Lagier JC, Parola P, et al. Hydroxychloroquine and azithromycin as a treatment of COVID-19: results of an open-label non-randomized clinical trial. Int J Antimicrob Agents 2020 ; 105949. doi : 10.1016/j. ijantimicag.2020.105949.

20. Netea MG, Domínguez-Andrés J, Barreiro LB, et al. Defining trained immunity and its role in health and disease. Nat Rev Immunol 2020. doi : 10.1038/ s41577-020-0285-6.

21. Domingo E. Mechanisms of viral emergence. Vet Res $2010 ; 41: 38$

22. Tripathy S, Dassarma B, Roy $S$, et al. A review on possible modes of actions of Chloroquine/ Hydroxychloroquine: Repurposing against SAR-COV-2 (COVID 19) pandemic. Int J Antimicrob Agents 2020 May 22 :106028. doi: 10.1016/j.ijantimicag.2020.106028.

TIRÉS À PART

J. Dubuisson

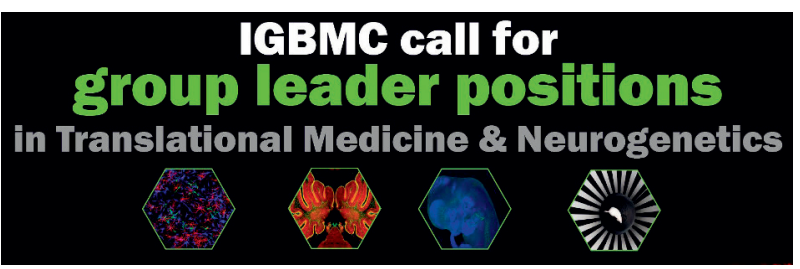

The Translational Medicine department (www.igbmc.fr) is welcoming applications for group leader positions (junior and senior) and aims to reinforce one of the following research axes:

- Neurological or muscle disorders with a focus on cellular biology, genetics, imaging, electrophysiology and/ or therapeutic development,

- Animal and cell (iPS, organoïds) models of neurological and/or muscle diseases,

- Bioinformatic and large-scale analysis dedicated to human pathologies.

Your application: Expression of interest will include, in a single PDF file, a curriculum vitae, a list of publications, names and contact of three referees, a brief description ( 2 to 4 pages) of past achievements and a detailed description (4 to 5 pages) of future research. Applications should be addressed to TMNcall2019@igbmc.fr subject: TMNcall2019.

The call is open until July $15^{\text {th }}$ 2019. Pre-selected candidates will be invited to the IGBMC to present a seminar and will be interviewed by the scientific direction of the IGBMC.

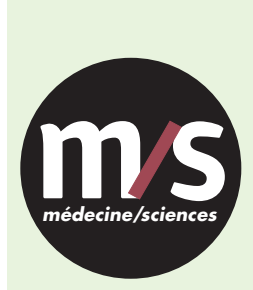

Tarifs d'abonnement $\mathrm{m} / \mathrm{s}-2020$

Abonnez-vous

à médecine/sciences
$>$ Grâce à $m / s$, vivez en direct les progrès des sciences biologiques et médicales

\section{Bulletin d'abonnement page 678 dans ce numéro de $\mathrm{m} / \mathrm{s}$}



